\title{
Postdigital Positionality as a Leader and Policy Maker
}

\author{
What Is Shaping the University and What Might the University Now \\ Shape?
}

Policy reviews will address the effects of the pandemic from numerous angles going forward to point to both existing and new inequities that Covid-19 has brought to light. Whilst this book has shared some of these examples, as contexts have unfolded, the main focus has been to encourage broad open debate on new policy discourse that will recognise the postdigital environment that universities now occupy and the diversity of human positionality within it. These theories together create a productive dialogue that might inform 'more inclusive' and critically sustainable inclusivity policy. As discussed already, universities are not contained institutions. Their activities are local, national, international, institutional and individual. All of these are aspects that are now also interconnected with what takes place in the virtual airing cupboard of the Internet. However, not all people in the communities surrounding universities have access to the Internet, to devices that might help them connect or to the skills that might enable participation.

An amusing, but also revealing illustration of the kinds of hybrid postdigital connections emerging across local government and the Internet, can be watched in a clip of a UK parish council meeting that went viral in early 2021 (YouTube, 2021). Posted online by people present, it was viewed on Twitter and YouTube by hundreds of thousands and also reported in national media, due to the altercations between those present. The video clip demonstrates the challenges of establishing points of order and regulations in an online meeting, as well as the different positionalities of those present attempting to also navigate the technology they are using. At one point a phone rings in the background and is answered during the meeting, whilst a form of cancel culture is enacted as the council chair is removed from the Zoom meeting by another councillor. Fierce objections from the vice chair ensue as he argues for the standing orders to be read. Requests to be respectful follow, with other members laughing in disbelief at how the meeting has unfolded.

This scenario is an anecdote worth reflecting on in relation to the local contexts and communities in which universities are located and the identities that 
HE institutions assume. Parish councils do not often hit the headlines, yet as the lowest tier of local government in different cities they play a valuable role in providing representation and consultation with district and county councils in matters such as planning, environment and recreation (Farrer \& Pidd, 2021). The sudden trending on Twitter of such a meeting is a powerful illustration of the reach of the virtual airing cupboard of the Internet, triggered through the necessity of meeting online during lockdown. In a further example of the challenges that can arise for community groups navigating online meetings in unfamiliar technology, an entire school board in California had to resign when a Webex meeting they believed to be private was publicly streamed. Members of the board made disparaging remarks about parents who were upset at school closures and quickly realised they had been heard and tweeted. A petition calling for their resignation followed (ввС Technology, 2о21).

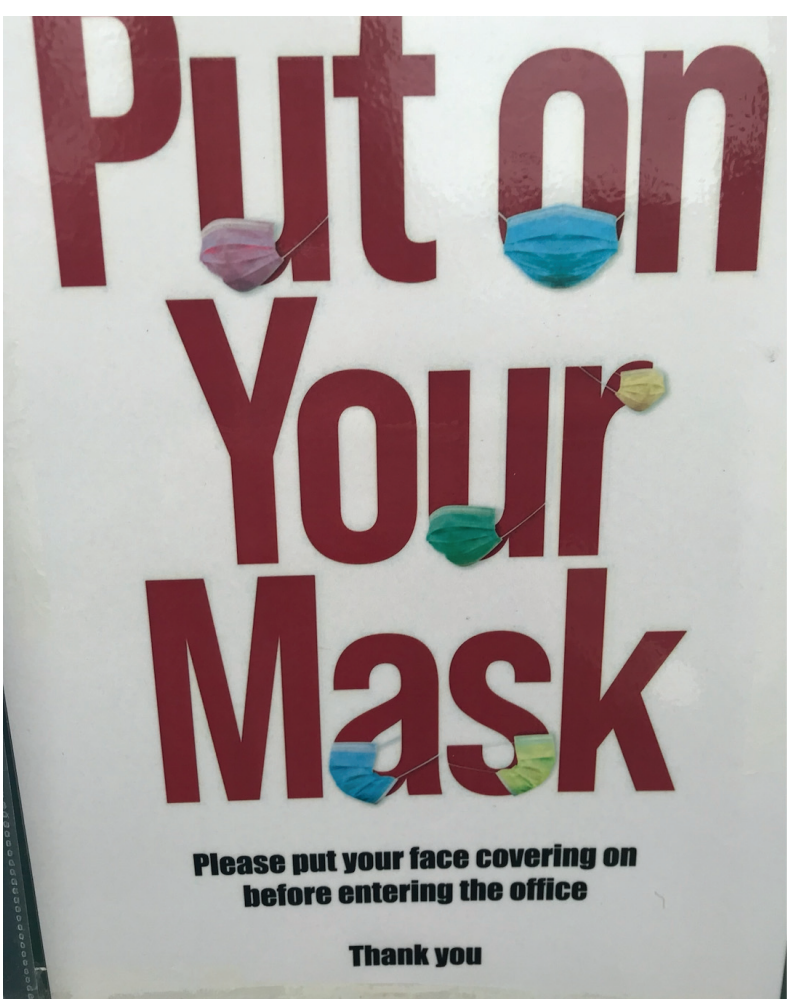

FIGURE 44

A reminder to 'put on your mask' before entering the office

In earlier chapters, this book pointed to some of these complex, postdigital interactions relating to the positionalities of students, teachers and researchers in this context, all of whom are also members of local communities and regions. The positionality of the postdigital university as an institution and 
that of its leaders located in hybrid learning spaces (Cohen, Nørgård \& Mor, 2020 ) is now the focus for this chapter.

In this introductory section to the chapter, I will set the context a little to discuss how the pandemic has revealed many underlying inequalities in the areas surrounding universities before outlining what the other sections will cover.

In a recently published Health Foundation report (2020), Build back fairer: the Covid-19 Marmot review, the pandemic, socioeconomic and health inequalities, a stagnation in health improvement in the UK is identified as a status quo that existed prior to the pandemic, that the country should not return to:

The levels of social, environmental and economic inequality in society are damaging health and well-being. As the UK emerges from the pandemic it would be a tragic mistake to attempt to re-establish the status quo that existed before the pandemic. (Health Foundation, 2020)

The report argues that 'this pandemic exposes the underlying inequalities in society and amplifies them' (Health Foundation, 2020: 5). On this basis this inquiry seeks to demonstrate effects from the pandemic and societal response on social and economic inequities, mental and physical health and their likely effects on health inequalities in the future. Yet in taking a socioeconomic focus, there are still only eight references to 'digital' in the main body of the report. All of these concern 'digital access' to services or 'digital exclusion' due to a lack of devices or infrastructure. Whilst digital access is a hugely important issue (not everyone can SmartShop as in Figure 45, or make online bookings as in Figure 46, or click and collect as in Figure 47) there are countless complex and deeply intertwined challenges that accompany digital access and the contexts of those who are disadvantaged (Traxler, Smith, Scott \& Hayes, 2020).

Understanding who will address these (frequently opaque) aspects of digital inequality is an ongoing issue for governments, universities, regional agencies, charities and all who seek to improve digital skills in communities.

The Not-Equal project (2020) discovered numerous challenges that digital ways of working throw up for already vulnerable groups. These include: safeguarding young people, a lack of moderation of their activities, patchy wifi connections, screen fatigue, online isolation, exhaustion from learning new platforms, physical issues, lack of skills and digital literacy, fear of technology, lack of trust, no consistent device if phones or laptops are swapped, shared, sold or stolen, exacerbation of existing mental health problems, a loss of intimacy, wariness of how to interact, privacy concerns, fears concerning protection and aversion to seeing one's own face on screen, or to being seen (Not-Equal project, 2020). 

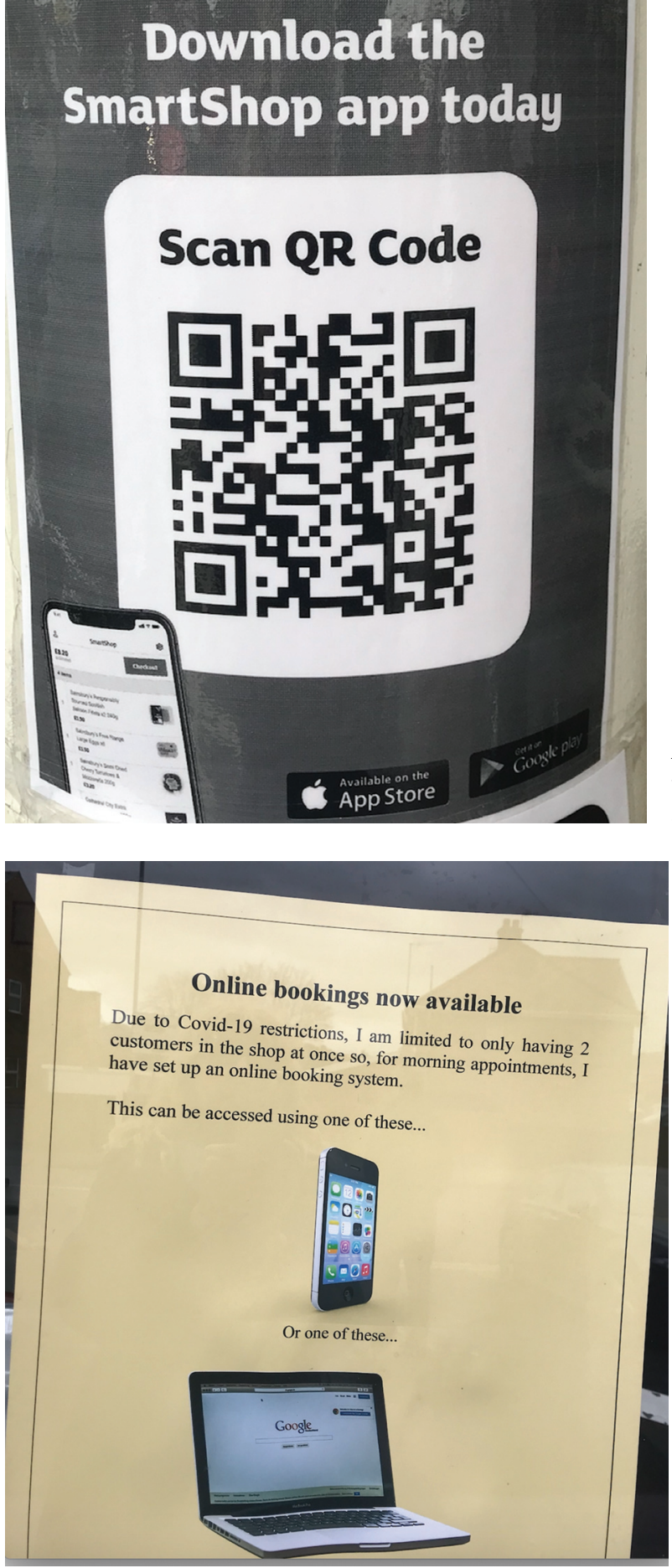

FIGURE 45

A reminder to download the SmartShop app to help avoid checkout queues
FIGURE 46

A sign indicating that due to Covid-19 an online booking system has been set up for appointments 


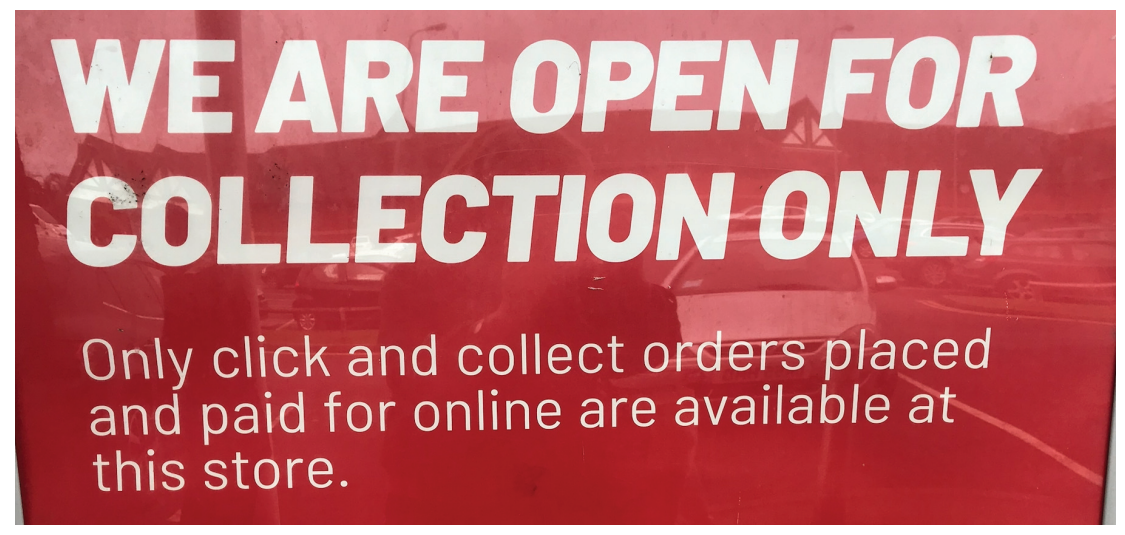

FIGURE 47 A sign reminding people that 'only orders placed online are available at this store'

All of these point to the individual and positional nature of the messy postdigital facets of our lives. Yet when digital devices or systems are mentioned in relation to inequality, diversity and inclusivity, university or government policy reports frequently omit these dimensions in an analysis that barely skims the surface.

In referencing issues of access to digital and online facilities in isolation, countless forms of bias, exploitation and discrimination are overlooked, alongside human agency, system legibility and negotiability (Mortier et al., 2020). Digital opportunities are understandably emphasised, along with skills and employability too, but it remains unclear as to who actually takes responsibility to attend to the many real but less visible aspects of digital life, that will still continue to hamper 'building back fairer'.

At the close of The Labour of Words in Higher Education, is it time to reoccupy policy? I explained why the current McPolicy discourse institutions still work to, is inadequate:

Attention needs to be paid to these documents. Not least because contradictory statements around social justice risk cancelling each other out, if widening participation and student retention are enacted by generic statements, projects and technology, rather than diverse staff and students themselves. (Hayes, 2019a: 150)

A persistent rational McPolicy discourse has been conducting its own form of cancel culture, by writing out the staff and students whose diverse labour constitutes the university, but also in omitting complex and far reaching digital interconnections with inequality and inclusion. Drawing on Ritzer's McDonaldisation thesis, McPolicy reached its point of irrationality then in a 
year when a pandemic revealed the 'contents' of the university for all to see. By contents, I refer to the humans and the 'more-than-humans' (Ulmer, 2017, Braidotti, 2019a, 2019b) in HE whose postdigital activities do not sit neatly within the walls of an institution. The virtual airing cupboard of the Internet connects and disconnects what people are and who they might become, in an epoch of broad and potentially irreversible digitalisation of life (OECD, 2O2O).

McPolicy closes down debate (Hayes et al., 2020). Or it did, until Covid-19 brought into the public domain the diverse and unique postdigital positionalities of students and academics across the globe (Jandrić \& Hayes, 2020a, 2020b, Watermeyer et al., 2020, UNESCO, 2020a). These testimonies that have flooded the virtual airing cupboard with a very different discourse to the sanitised impression of what happens in university activities that is generated in McPolicy. So, in a sense, my call below has already received a unanimous response:

It therefore is time for staff and students, as a collective community, to protest this language and change the way in which divisive forms of $\mathrm{HE}$ policy are written. (Hayes, 2019a: 150)

Rational approaches in McPolicy omit these distinctive and varied aspects of who each of us are, as individual people. They omit the communicative powers concerning knowledge that are now afforded to the wider population in the virtual airing cupboard. Thus, they omit the global citizenship we each hold alongside any institutional membership. A generic, repetitive McPolicy reflects the consumer culture of our current political economic structuring of society. It is unsustainable though to represent a new political bioeconomic discourse that our global society now seeks (OECD, 2009, The Bioeconomy Council, 2019, UNESCO, 2020d).

Linking positionality with postdigital theory yields a broader understanding too of why these concepts need to remain in continual dialogue to inform inclusivity policy in this context. University policy documents and frameworks for inclusivity currently fail to acknowledge the dialectical nature of human activity, computer systems, learning, research, data, material and biological change. The technological and cultural split already discussed can be noticed clearly as some policies home in on matters of social justice and human rights but omit any reference to the ethical issues that are ingrained in our data gathering and data-driven technological platforms across the Internet. Meanwhile, policies that urge a use of technology tend to frame this in the weary arguments of best practice, an unquestionable enhancement of the student experience and a measurement of excellence. In a wider global context this 
separation of policy interests that concern cultural issues, from those that concern technological ones, tends to serve a variety of commercial interests. It does not though adequately serve an emancipatory inclusivity agenda in universities. Reflecting on educational reform from a global perspective, Saltman and Means (2019) highlight trends that are converging to fundamentally alter education from a democratic to a commercial endeavour across the world:

Transnational institutions, such as the Organisation for Economic Cooperation and development (OECD), the World Bank, the World Trade Organisation, and powerful transnational corporations, such as Pearson, are promoting an interconnected set of global educational reforms that seek to align national systems of education with the demands of transnational capitalism and elite economic and political interests. (Saltman \& Means, 2019:1)

Given this situation, where many universities have now invested in corporate partnerships on the grounds of improved efficiencies and rational ideas of progress, there are some key choices looming for leaders and policy makers in relation to AI, algorithmic activity, data, cloud ethics (Amoore, 2020) and equality, diversity and inclusivity agendas. As discussed earlier, we cannot inhabit only the parts of 'the cloud' we choose to, as individuals or as institutions. Nor can we easily track down accountable humans outside of algorithms to hold them to account when 'algorithms are implicated in new regimes of verification, new forms of identifying a wrong or of truth telling in the world' (Amoore, 2020). Such a situation now requires university leaders to revisit their whole mission as sites and producers of knowledge, when they are no longer the main users of knowledge (Delanty, 2001: 152). Furthermore, that knowledge is inextricably intermingled with information and data that has huge commercial value. The increased instances of cyberattacks, ransomeware and phishing emails targeting universities further attest to this (Afifi-Sabet, 2020, Hellard, 2020).

Rather than write an inclusivity framework and trust that 'the principles of inclusive practice are well established' (Department for Education, 2017), there is a need to confront what is constantly being re-worked in the cloud to understand how this alters how inclusivity is actually experienced by individuals. Huge corporations now control human online identities. Such control does not cease when students or staff log off from a computer or a phone. Our datafied selves continue to be shaped, altered, celebrated, exploited or cancelled, but nor do they disappear. Deeply personal facets of our identities, including race, gender, disability and religion are 'described by 'ones and zeros' and 'defined by templates of data' (Cheney-Lippold, 2018). Furthermore, 'algorithmically 
produced categories replace the politicised language of race, gender and class with a proprietary vocabulary that speaks for us' (Cheney-Lippold, 2018) and this is speaking to commercial monopolies, that have different values to universities. This then severely hampers the ambitions of any university committee to 'embed' or 'mainstream' practices for inclusivity which do not remain static. A new approach is needed that plays to the strengths of universities, rather than one that simply exposes their weaknesses in the corporate playing field. Here universities are faced with decisions on claiming their own postdigital positionalities and shaping their own response as leaders in this field, before becoming simply additional resources for the big commercial monopolies.

Peter Scott (2018) has argued that 'the contemporary university is subject to two (apparently contradictory), forces'. He describes firstly:

the drive towards the modernisation of its governance and management, often in ways that reflect corporate structures more familiar in the private sector (and other parts of the reformed public sector). This drive has been accompanied by the growth of performance management, both of institutions and individual teachers and researchers; the more prescriptive identification of goals and targets (at the expense, perhaps, of traditional notions of autonomy and academic freedom); and more explicit - and intrusive? - forms of audit, accountability and evaluation. (Scott, 2018: 35)

I demonstrated in my analysis of McPolicy how such rational forms of governance have come to be represented in strategic discourse. Common sense statements suggest that technologies, policies and institutions, not people, have the autonomy to 'act' to accomplish all aspects of academic work. If people are left feeling 'insignificant' (Leone, 2020) then perhaps this is happening to institutions too. Scott then describes the second force as:

new modes of learning, a more open curriculum and more distributed patterns of research. Examples include the popularity of (technologyenhanced) self-directed learning, the growth of massive open online courses (MOOCs), the spread of open-source publication and greater emphasis on the impact and application of research. (Scott, 2018: 35)

As a former Vice Chancellor, Scott's argument is that whilst both of these forces reflect deep-rooted changes in the nature of modern higher education:

it would be misleading to see them as always or inevitably in conflict. However, they do pose new dilemmas about how to maintain an appropriate 
balance between the necessary management of the large, complex and heterogeneous organisations that modern universities have become and their capacity for innovation and creativity. (Scott, 2018: 35)

Thinking about this analysis in relation to the broad observations on a need to re-link cultural reproduction and technological reproduction in $\mathrm{HE}$, Delanty discussed a market-driven capitalism 'shaping the university in the image of technoscience', as disengaged from 'battles of cultural identity' (Delanty, 2001: 157). Nearly twenty years later we are in the grip of a pandemic that has revealed just how closely interconnected these economic, technological, cultural and biological battles really are. The underpinning economic focus on productivity has in recent decades replaced an 'open intellectual enquiry' (Olssen \& Peters, 2005) through which we might debate postdigital connectivities. Complex intersections across the virtual airing cupboard now necessitate this open dialogue when inclusivity is the focus. Davies (2016) discussed different phases and policy dynamics of neoliberalism over recent decades that have sought to 'prop up' the broken model of capitalist accumulation (Davies, 2016: 133). In a sense these mutate in a fashion analogous to different strains of the Covid19 virus, which also disadvantages the vulnerable. What remains a problem is that persistent rational approaches omit the distinctive and varied aspects of who each of us are and the variable positions we find ourselves in. They also omit the communicative powers concerning knowledge that are now afforded to much of the wider population.

However different authors choose to frame these arguments, there seems to be some broad agreement that tensions between corporate structures, compliance and prescriptive targets for excellence have driven divisions that could hamper creative approaches deeply connected with our human cultural identities. Our current HE policy discourse structures set out the boundaries of what staff and students come to believe is possible, even if as individuals they do not closely engage with the processes of policymaking themselves. The related narratives and arguments of McPolicy make their way into all of the realms of activity in universities and resisting such dominant rhetoric becomes challenging.

As discussed in Chapter 2, postdigital positionality and the twofold debate this book is concerned with for inclusivity policy, centres around the interactions, back and forth across humans and machines in personal, individual contexts, and across interpersonal relations. AI has enabled machines to mimic the cognitive functions that humans use, whilst interacting with humans. How this is experienced is unique for each person and thus also significant to how leaders respond towards inclusivity in postdigital HE. This is because multiple 
and diverse 'actors' (that are not all human, and not all machine) are now altering much of what is still being described as 'established' or 'inclusive' practices in universities.

Scott (2018) points out that tensions and even contradictions between compliance and creativity are not though inevitable. He argues for systems of coordination and balanced solutions where creativity can be nurtured and strengthened, pointing out that this is:

essential to the healthy functioning of universities in the twenty-first century because of the complexity and heterogeneity of their missions. (Scott, 2018: 46)

Work undertaken by Adrianna Kezar and Jaime Lester (2010) suggests that positionality theory expands knowledge concerning leadership. This is because too simplistic an approach can often be taken towards the role played by the social identities of leaders. Policy rhetoric that imposes broad categorisations on human beings tends to uphold the image of leaders in static positions from which they take rational decisions based on common sense. The placing of people into any kind of grouping objectifies the human qualities of this group. This can translate into linguistic constructions in policy that also attribute actions and feelings to this socially constructed category of people. Positionality theory points instead to multiple overlapping identities (Kezar, 2002: 96). Kezar \& Lester argue that our understanding of leadership in an HE context remains partial without taking into account features of identity such as race, disability or gender.

Postdigital positionality theory can expand this approach further, to reconceptualise leadership and policymaking by including people's 'postdigital identities'. These have a 'dialectic relationship' (Freire, 2000: 50) with the other key elements of our existence and how we each perceive ourselves as individuals. This takes into account the complexities of postdigital experiences as part of the multiple identities that affect leaders and the processes of leadership. There are now intricacies arising to show that statements like 'the principles of inclusive practice are well established' (Department for Education, 2017) are misconceptions. The principles of inclusive practice instead require a dynamic ongoing dialogue. Prior (2020) points however to the problem that 'there is still sector-wide ignorance surrounding how to deal with knowledge that is subjective and embodied' (Prior, 2020: 188). Yet this is also knowledge that is a crucial aspect of any inclusivity programme, if equality and diversity are to be celebrated and if marginalised groups are to be supported to recognise opportunities. 
In the remainder of this chapter, rational conceptions of leadership in $\mathrm{HE}$ will be examined firstly, in terms of the current positionality of universities in postdigital society. As complex marketised and multi-layered institutions where McPolicy is generated, this form of discourse is culturally distant from individuals and their academic identities. An examination of cultural forces at play within universities cannot sit apart from individuals, or from the global and transnational technological advances already discussed. The work of multinational corporations and alliances now shape our lives based on the data we have become in the cloud (Ball, 2020, Cheney-Lippold, 2018, Amoore, 202O, Selwyn, Macgilchrist \& Williamson, 2020). This data is deeply personal, it relates to our identities and perceived possibilities of who each of us might become as diverse citizens and human beings. Komljenovic (2020) argues that universities have tended to focus on data privacy issues which are important, but from a political economic perspective, the data value models of commercial ed tech companies need our attention too. These are quietly restructuring cultural and technological values in HE.

The implications for inclusivity policy in institutions are too huge to ignore, or to allow cultural concerns to be raised independently of the technological ones. Then there is the question of control. Whilst universities attempt to control and embed inclusivity agendas, as if these existed apart from digital advances and new educational technologies, their partnerships with major commercial players are also bringing new consequences for inclusive practices in relation to data driven systems. The ongoing privatisation and commercialisation of education has rapidly advanced across the globe during the 2020 pandemic. Leaders in governments and universities alike were ill-equipped to catch up with the rapid technological changes in postdigital society and so they relied heavily on the advice of corporate business partners. These companies are not disinterested advisers though when it comes to data. They take rational, solution-based and profitable decisions. Therefore, in a sense this is not unlike trusting foxes to take care of the hens. The values and intentions of the parties involved are somewhat different. It is also a blurred area too where wealthy philanthropists have funded private technology companies to become embedded in public education systems bringing long-term reforms (Hogan \& Williamson, 2020). This provides new powers for technology consortiums to influence educational policies but based on values of investment and profit. It places considerably less emphasis though on the interests of emancipatory education that seeks to bring opportunities for knowledge to those on the margins, in terms of levelling up.

The second half of this chapter is concerned with the responses that are open to university leaders and policymakers. These require a reflexivity that 
is a key strength of universities to be applied in re-shaping how institutions are positioned in postdigital society. Rather than seek to control aspects of the virtual airing cupboard by embedding them inside the structure of the institution, universities are re-visualised as centres of critical, cross-sector postdigital dialogue. In this vision the university, as a key producer of critical forms of knowledge, becomes more of an arbiter of postdigital communication, not another bystander looking on. In this role there can be an acceptance that inclusivity agendas are not simply regulated as if they lived inside the university, when so many powerful societal forces now act upon these. Therefore, the future of the university lies in preserving 'relatively non-institutional space' and 'seeing the university as the site of reflexively constituted knowledge' in order to 'appreciate its role in contemporary society' (Delanty, 2001: 155). This a perspective that treats digital skills and online activities as every bit as 'real' as place-based opportunities for those in disadvantaged situations. It is a positionality that can engage with the notion of 'critical sustainability as part of a post-corporate cultural model and as an alternative to the neoliberal conception of sustainability (Delanty, 2020: 1). Therefore, rather than acting as a resource for corporate business to simply mould, the university becomes instead, a critically reflexive authority at the intersection of powerful crosssector, postdigital debates.

\subsection{What Is an Institution?}

Much research has been undertaken on ways of understanding institutions and organisational structures. There have been recent attempts to consolidate these ideas too in the light of social change and to examine institutional structures, processes and interplays at the world system, transnational, societal and industry levels alongside micro forces (Scott, 2010). In terms of the concept of postdigital positionality, this is an emerging debate that can also be examined at micro, personal and interpersonal levels right through to macro transocietal levels.

Reeves (2019) argues that:

Framing an organisation as a complex adaptive system shifts focus away from the individual and towards the organisation as the unit of analysis, and such a shift is consistent with the anti-reductionist rhetoric of postdigital to move beyond a conception of an organisation as something that can be reduced to individual people, resources, and data. (Reeves, 2019: 156)

Increasing human agency to shape organisational evolution is argued to be dependent on a reconceptualisation of value. This requires a more conscious approach to determining what is important in the activity of organisations 
(Reeves, 2019: 156). As institutions, universities tend to describe their top-level mission statements and strategic plans and ambitions. The policies that sit beneath this overview may or may not articulate well with it. As I have shared my findings on McPolicy from The Labour of Words in $H E$ at different institutions I have consistently been told by staff and students that they do not recognise themselves in the policy discourse of their university. They view it as disconnected from them personally and from their academic activities. This is not therefore a good starting point for inclusivity policy to be cast in the same vein. Scott (2010: 6) defines institutions as:

Social structures that have attained a high degree of resilience [and are] composed of cultural-cognitive, normative, and regulative elements that, together with associated activities and resources, provide stability and meaning to social life. (Scott, 2010: 6)

This suggests that individuals are deriving meaning at different levels that do not sit neatly in one element of organisational management. Rather there is an interdependence across the diverse elements, making it important to look at these holistically and reflexively, in terms of positionality, rather than rationally and objectively. Scott describes three interdependent elements of organisational management as:

- Regulative: these elements are formalised and rational, easily planned and strategically manipulated, with a need for clear directives, alignment of incentives, and surveillance. Regulative elements, if not supported by cultural beliefs, are more likely to be evaded and behaviour of staff becomes decoupled from the rules and formal structures.

- Normative: actors are seen more as social persons who care deeply about their relations to others and adherence to the guidelines is motivated by their own identity, personal ties and informal relations with co-workers.

- Cultural-cognitive: elements are socially constructed symbolic representations that frame individual perceptions and decisions. Deeply entrenched assumptions and conceptions of the 'way the world is' are likened to culture as 'the software of the mind' (Hofstede, 1991). Cultural-cognitive elements provide the bedrock for normative prescriptions and regulative controls, because norms and rules must refer to institutionally constituted entities (Scott, 2010: 6).

Scott argues that this 'three pillars' framework (that many HE institutions often structure a strategic plan around) becomes emasculated if not closely connected to human and physical resources. As such it is necessary to be aware of: institutional variety, the interdependence of and interactions between 
organised units at multiple levels, the effects of non-local, as well as local factors, appreciation of the temporal nature of social life, the ongoing effects of the past on the present and future, to emphasise the importance of ideas that mediate between environmental conditions and actions to promote diversity, and new types of social behaviour and novel systems (Scott, 2010: 18).

This understanding sits well with the ideas from Reeves on the 'anti-reductionist rhetoric' of the postdigital (Reeves, 2019: 156). However, there seem to be few forums where the identity of the university might be critically examined and reimaged outside of the constraints of institutional McPolicy.

\subsection{Bureaucratisation, Rationality and Positionality}

Ginsberg (2011:25) points to the challenges that have emerged from a rational bureaucratisation of $\mathrm{HE}$ but one that has irrationally failed to free up time for academic staff 'to dedicate themselves to their main tasks, teaching and research' (Jandrić \& Hayes, 202od). Universities are filled with:

armies of functionaries - the vice presidents, associate vice presidents, assistant vice presidents, provosts, associate provosts, vice provosts, assistant provosts, deans, deanlets, deanlings, each commanding staffers and assistants - who, more and more, direct the operations of every school. Backed by their administrative legions, university presidents and other senior administrators have been able, at most schools, to dispense with faculty involvement in campus management and, thereby to reduce the faculty's influence in university affairs. (Ginsberg, 2011: 25)

Further irrationalities have emerged as the layers within universities have become more complicated to meet the demands from regulatory bodies, such as audits of data on student access, participation, retention and progression (Office for Students, 2018, 2019). Whilst all of these topics are important, a narrow focus has been adopted for inclusivity in this context. It has largely engaged with the humans concerned, and rather ironically has done so, through engagement with data, not people. Furthermore, in removing faculty from central institutional administration including policymaking, it becomes hard to argue that faculty do not engage with policy. Meanwhile members of faculty can find that they still spend many hours on the layers of local administration that still exist in their department (Jandrić \& Hayes, 2020d).

\subsection{How Might He Policy Pivot to Postdigital Policy?}

Teachers are urged to continually improve on what they teach in $\mathrm{HE}$, researchers are urged to improve the impact from what they publish, but this is not 
a discourse heard very often in relation to policymaking processes and the related discourse that is written up. Scrutiny in this area seems to have been missed off the quality assurance and enhancement grid, as I don't remember noticing The Policy Excellence Framework (the PEL perhaps?) being devised to join the other REF, TEF and KEF frameworks that UK universities are required to work to. In The Labour of Words in HE I observed that:

These are the decades in which academic labour has been increasingly, rationally audited for quality and performance (Shore \& Wright, 1999; Ball, 2003). Yet somehow, rather irrationally, we have failed to quality assure or enhance, the very language of McPolicy in which we write our strategies for enhancement. (Hayes, 2019a: 6)

Policy writing committees could be more inclusive (and creative) given the shift during the pandemic to write university policy via online systems, rather than gathered around a table on campus. A focus is heavily placed on how teachers might flip a classroom and pivot to online education, but where are

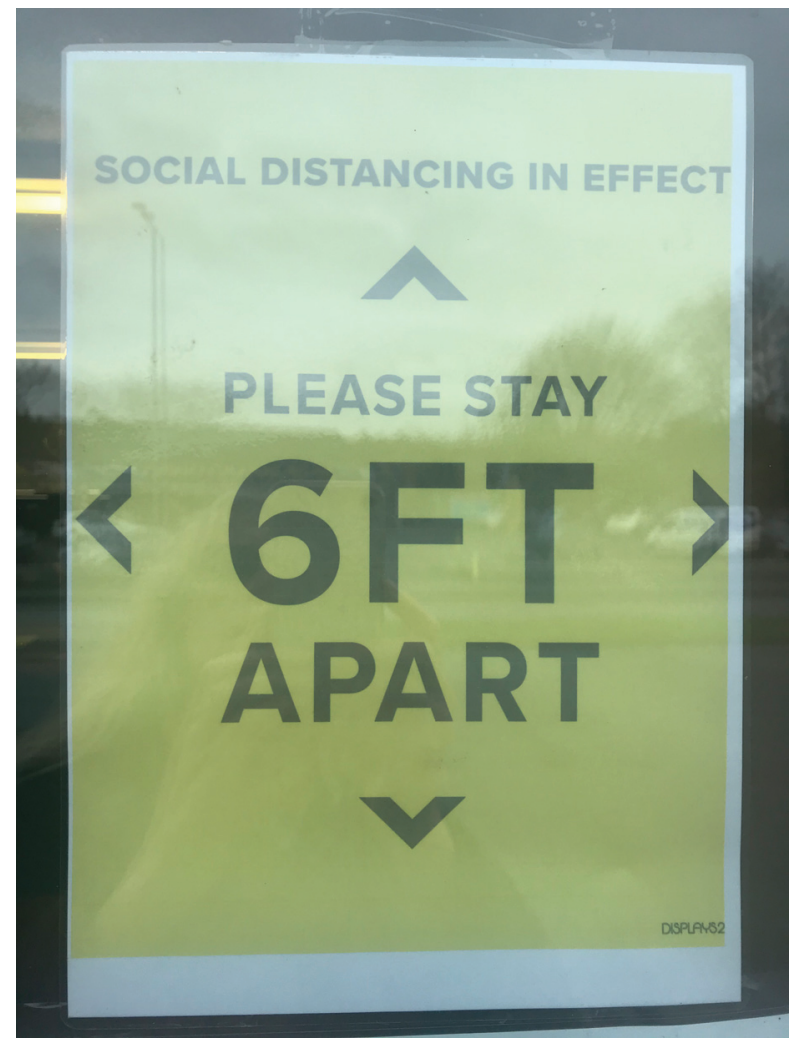

FIGURE 48

A sign instructing people to please stay apart from each other 
the discussions for opening up creativity in policy making, now that there are captive experts and audiences, just as there are for online conferences?

In recent decades universities have omitted a crucial part to policy making and policy writing. Indicative of a productivity-driven, neoliberal approach across all of academia perhaps (particularly since student fees were introduced), the broader dialogue that would reflect the 'sociocultural dynamism of policy processes' (Nudzor, 2009: 503) has been bypassed. Instead, the rational problem-solving approach has dominated and the documents we have to show for it (closely examined in The Labour of Words) have been in social isolation from each other since long before Covid-19.

Yet, even lonely HE strategies aimed at solitary topics were constituted within a context (despite a use of language that frequently omits this background). They demonstrate (if we really take time to look) what HE policymaking had become prior to the pandemic, a set of socially distanced topics that persist in addressing cultural issues in isolation from technological ones (Delanty, 2001).

Opening a dialogue now concerning the new possibilities before universities, where they might take into account the dynamic personal postdigital context around individuals and reflect this in policy, would offer a liberating and potentially more effective route forwards. If any further evidence were needed as to why such dialogue is necessary, then much can be sourced from the post-pandemic debates already taking place at this time of writing (Carrigan, 2020a). Given that policy is neither a problem-solving tool, nor a process, but both (Nudzor, 2009), there is time yet to shape a dynamic 'new normal' for $\mathrm{HE}$ policy discourse. One that draws more closely on a global focus on sustainability. This could soon show us what a more ecological, political bioeconomic discourse looks like, rather than a political economic one which emphasises only productivity and consumption.

\subsection{Digitalisation, Convergence and the Need for a Shift in University Policy}

The technological and scientific convergences that are described in recent reports on digitalisation have far reaching consequences for what we do in $\mathrm{HE}$ both globally and locally that 'cannot be overstated' (OECD, 2O2O: 20). Digitalisation runs deep and it is happening quickly therefore university leaders have a challenge before them as to how exactly to engage with this in policy. There are European plans to 'overhaul' the digital market too, including how tech giants operate' (в вс Technology, 202о). This is aimed at addressing a dominance that big players like Google and Facebook have assumed. In particular their use of the data they have gathered from one service that is used to 
'improve or develop' a new one in a different area, making it difficult to compete with them. They have become 'gatekeepers' who have set the rules of the game for their users and their competitors (BвC Technology, 2020). Ball (2020: 232) argues that we 'need to work out how to treat personal data now it is so valuable'. He suggests though that this may require many smaller fixes rather than one big one if we are hoping for sustainable change.

Universities could also adopt this smaller fix approach and feed into different policies, each of the various related compliances that legislation requires, but there are broader responsibilities for university leaders to consider. These go beyond the regulative elements identified earlier by Scott (2010). There are also normative and cultural-cognitive elements to deliberate, if the university is to consolidate its positionality in postdigital society. University leaders will need to take debate well beyond what Williamson refers to as 'political computational thinking' where assumptions are made that 'policy problems can be addressed using the right code'. In this model students are inculcated into 'the material practices and systems of thought associated with computer coding' and encouraged to become contributors to new forms of digital governance (Williamson, 2016: 39). However, all this does is shift responsibility elsewhere rather than confronting more fundamental inequalities that exist in the digital realm. Instead universities need to be ready to link probing concerns about digitalisation and sustainability, with inclusivity policy, which requires a fundamentally different discourse.

\subsection{Convergence of Global and Local Policy Dialogues}

It is also a discourse where there is much to be learned at both global and local levels from interventions during the pandemic. This is demonstrated in a recent research project that was conducted by a team in the Education Observatory, at University of Wolverhampton (Traxler, Smith, Scott \& Hayes, 2020). The research, which was commissioned by the Department for International Development (DFID) EdTech Hub was intended to (1) identify digital ideas, in whatever form that might maintain the continuity of education systems and (2) that might stop existing or potential disadvantages being amplified or exacerbated by Covid-19 or by the responses to it. The report which emerged from this project is based on a large-scale review of literature, from both academic sources and non-academic sources, on consultations with specific groups of experts and policy makers and on material and ideas from a wide network of contacts and collaborators (Traxler, Smith, Scott \& Hayes, 2020). The relevance this research has for new approaches towards HE policy discourse arises from its deeply dialogic and dynamic approach towards understanding the positionality of those in crisis and their postdigital contexts across the globe. 
As mentioned earlier, HE policies that self-isolate from each other within institutions are a problem because they fail to connect all of the factors affecting inclusive practices, particularly in overlooking postdigital factors and individual positionality. However, the practice of universities simply reproducing similar policies on different topics is also a trend that risks these documents being meaningless to those they are aimed at. Now that the Covid-19 crisis has revealed how so many problems arising in the Global North have already been experienced and addressed in creative ways across the Global South, there is much to be learned from how other nations are developing inclusive practices. Research publications from the Global North have dominated knowledge about $\mathrm{HE}$, due to the underpinning global economy on which universities have largely based their research and policy making. These papers and indeed the related policies should not be read in isolation from those bearing insights on crises from the Global South because Covid-19 has presented many similar challenges to those already being tackled in the developing world. Steps to improve access and continuity of education for marginalised groups through technologies in Low to Middle Income Countries (LMIC) hold relevance for disadvantaged groups living in local regions of developed countries. The Learning through the Crisis report (Traxler, Smith, Scott \& Hayes, 2020) explains how amongst the research methods undertaken, a Delphi process and ideas from a wide network of contacts and collaborators informed policy recommendations. The Delphi method is a systematic means for a panel of experts to respond to questions and share views (Brady, 2015, Okoli \& Pawlowski, 2004) which was adapted in this research to gather opinion via Google Forms. This enabled rapid insights to be shared amongst policy makers from different countries alongside those teaching, researching and supporting the use of digital technologies and analogue interventions, to address disadvantage. In this way, the individual lived experience on what has worked and what hasn't in contexts of crisis was brought into dialogue. There are many ways that Delphi can be adapted as a process to support more dynamic and inclusive forms of policymaking using online systems across different cultures and communities.

Our own adaptation of Delphi demonstrated that dialogue is crucial in the policy making decisions to address crises. Digital technologies make these exchanges possible now in much broader contexts to develop new approaches towards inclusivity. Those who participated in the Delphi exchange with us were working with different minority groups or were specialists in different regions of Africa. Others had considerable experience in distance learning or expertise in policy making in different regions or with marginalised groups. More detailed explanations of the Delphi process (which was really a digitally-enabled simulation of a face to face discussion) can be read in the report. At a time 
when many university staff are already communicating across online forums to conduct their daily activities, the chance to involve local regional agencies or international ones to inform policy on inclusivity has never been more relevant. This allows an honest approach to towards including human (and technological) experience in policy making rather than simply writing about a package that universities are claiming to 'enhance' called 'the student experience'.

The simplistic McDelivery approach to policy not only treats students as if they are on a production line, the policy texts themselves then suggest that 'the student experience' has power to act on behalf of both students and academics alike (Hayes, 2019a: 6).

\section{$2 \quad$ Ecological Approaches towards Policy That Begin from Positionality Not Rationality}

Beginning to address matters of inclusivity from any point of rationality needs to be questioned as it is only one possible starting point. In a recent publication entitled Incalculable experience (Bertelsen, 2019) the importance of positionality with regard to experience is expressed. The starting point here is that rather than a rational measuring of student experience, such 'pre-determining pretences of the neoliberal education system' should be resisted in favour of exceeding 'the count':

Thought and research, reading and writing are activities whose value should never be determined in advance. Neither should their value be reduced to the post-determinations of metrics. (Bertelsen, 2019)

Currently universities seek to control each of the various challenges that present themselves from 'outside' by placing them within rational frameworks that measure pre-determined ideas about inclusive practices or employability and what counts as 'success'. To move beyond a simple calulative model that sat within the current neoliberal political economy model and took a mostly regulative approach, a more critical dialogue is needed for the normative and cultural-cognitive levels to strengthen the postdigital university. Neither inclusivity nor sustainability agendas can be simply regulated, as if these lived inside universities when so many powerful global forces now act upon these areas of life. There is though, a choice for university leaders to take between either attempting to treat the sustainability agenda as if it were just another in the line-up of corporate policies or adopting a more critically reflexive stance in a 'post-corporate cultural model' (Delanty, 2020: 1). 


\subsection{Critical Sustainability}

If the university adopts a more ecological approach then 'critical sustainability as part of a post-corporate cultural model' provides one 'alternative to the neoliberal conception of sustainability' (Delanty, 2020: 1). This would provide a different route for universities from their current purpose which has tended to see them reduced to something of a resource for corporate businesses to mould and a supply system providing work-ready graduates for companies to employ. Given that digitisation is promising to fundamentally disrupt current understandings of humanity itself, the university has responsibilities as a critically reflexive facilitator located at the very intersection of these powerful cross-sector, postdigital debates.

At a time when education is being placed as central to the United Nations Educational, Scientific and Cultural Organization roadmap for sustainable development (UNESCO, 2O2Od) and global activities towards sustainable futures (The Bioeconomy Council, 2019), Delanty argues that 'a clear relation to critique' has not yet been established. He points out that there are good reasons to be critical when sustainability derives from a 'global policy and corporate discourse' that is 'replete with contradictions and ambivalence'. Citing a fundamental problem (that I would argue sits well alongside the sorts of irrationality of rationality described by Ritzer), he suggests that:

the notion of sustainability seeks to sustain that which has now become unsustainable and is therefore incompatible with radical political ecology and a concept of nature in keeping with the age of the Anthropocene. (Delanty, 2O2O: 2)

Delanty contests that it is only when the idea of 'unsustainability' is brought into the picture that the notion of 'sustainability' takes on a wider significance, in responding to calls for alternatives to endless growth and the widely felt need for a more collective responsibility. Whilst there is consensus on the need in general for sustainable environmental policies, in terms of 'what needs to be done', there is little consensus on 'how it should be done'. In proposing a post-corporate cultural model as an alternative to a neoliberal conception of sustainability, this also offers something of a corrective to ideas of 'post-sustainability' (Foster, 2017), 'namely the argument that sustainability has become devoid of relevance and has become an empty term' (Delanty, 2020:3).

Whilst sustainability, like so many other buzz terms has been made 'banal' in corporate discourse, with multiple meanings concerning what is to be sustained and by whom, 'the term has now become integral to the very nature of 
democracy, such that it no longer resides in a domain separate to social and political institutions and practices. To this end Delanty argues that 'sustainability is an inherently cultural category and needs to be understood as such' (Delanty, 2020: 4).

\subsection{Cross-Sector Postdigital Dialogue Led by Humanities and Computing} The arguments above, coupled with Delanty's (2001) call for a reconnection in universities of the discourse between technology and culture, offer productive routes to think through future possibilities for ecological pathways for university policies. However, these then require a dual resistance from both humanities and computing, to totalising McPolicy agendas. They need to be widely inclusive too, of cross-sector postdigital dialogue.

So how might humanities and computing together lead such a debate? Michael Peters argues that currently we find ourselves in a 'bad ecology', a storm that 'links data, life and the planet', it:

Integrates human beings into systems as part of the hive intelligence that harnesses big data and puts its analysis to new commercial uses. (Peters, 2020: 593)

Computing therefore has a place and a ready supply of data, but as discussed in Chapter 2, there are probing HCI questions and complex intersectional debates already taking place. In the humanities on the other hand there is also broad debate across different branches, from digital humanities to philosophy, but perhaps it is important to re-examine what remains in the former categories of thought:

Meanwhile, philosophy is still busy debating its age-old questions: freedom, agency, happiness, the good life. (Peters, 2020: 593)

At this point of crisis where the information age is meeting new bioinformational and bioeconomic challenges and possibilities, Peters points out that:

Philosophy in the age of information has gone off-shore to the AI engineer. The old categories are empty containers formulated in the Enlightenment and form an ideal board game with no winners. They are not able to describe or analyse the current fifth generation cybernetic system rationality that engulfs us all changing the very conditions of existence. (Peters, 2020: 593) 
Therefore, the question of positionality is key here, if the fields of humanities and computing are to jointly lead a cross-sector postdigital debate, particularly one in which our human biological status itself is in the process of being digitised.

\subsection{Academic Freedom of Speech in the Postdigital Debate}

Meanwhile, on the topic of postdigital debate there are also issues for universities who don't rise to the challenges of taking such a dialogue into the wider community. Dorrell (2020) picks up on the risks that arise for academia in failing to engage with the broader politics and agencies outside of institutions.

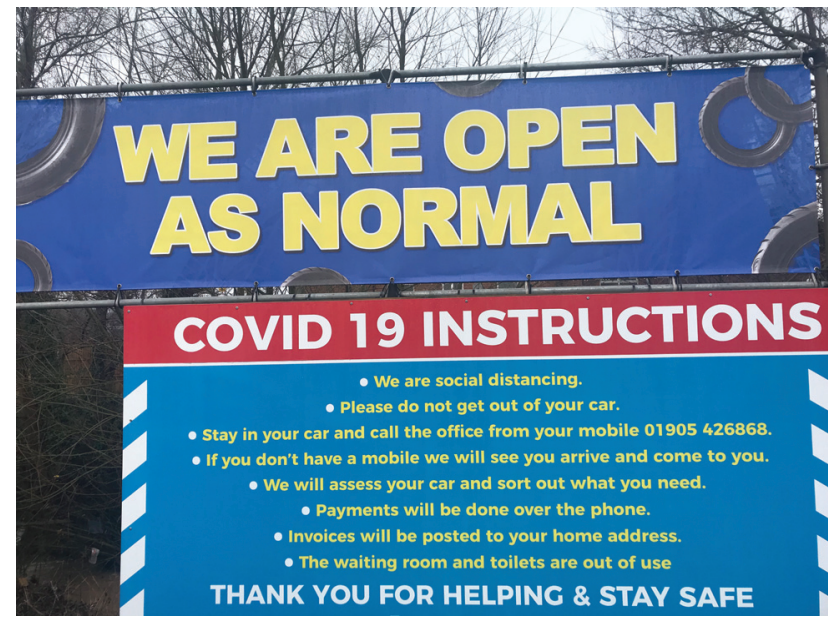

FIGURE 49

A local business details its policy for operating as 'normal' during the Covid-19 pandemic

On the issue of 'academic freedom of speech' he perceives there are 'escalating culture wars'. Referring to a report by a particular think tank and adviser to government he suggests that:

If universities can make themselves central to this conversation, then they might no longer be seen as an enemy to defeat. (Dorrell, 2020)

All the more so, 'if universities place themselves at 'the centre of the conversation about improving the lives of those who live in the satellite towns beyond their immediate horizons' (Dorrell, 2020). This includes building strong connections with local government and the many businesses that have struggled through the pandemic lockdowns (Figure 49).

Thus, universities who engage with the place-based agendas of 'levelling up' (University Partnerships Programme Foundation, 2018) will be in a better position to lead cross-sector debate. However, this also requires a stronger focus on 
local priorities and not just on institutional ones. It is only through a stronger connection with citizens themselves that universities will be able to develop their postdigital inclusivity agendas to become active and meaningful.

\subsection{University Strategy and Postdigtial Organisational Change}

University strategy therefore cannot be based only on lessons learned from the past or on solutionist views of digital technologies to simply 'enhance' what $\mathrm{HE}$ already does. The shift taking place as life itself becomes digitised and a convergence of disciplines follows could provide us yet with new critical and sustainable routes out of the crisis. Reeves suggests that it is not enough to 'place a passive trust in technologies that we barely understand', but in taking a postdigital perspective on organisations, we might 'prioritise the experiences that we want organisations to provide'. This concerns what is valued 'for society and the environment' but we need to adapt our behaviour accordingly' (Reeves, 2019: 197).

Meanwhile, Scott (2010: 17) points to a need to:

consider the full range of mechanisms that allow institutional forms to emerge, the struggles over their shape and meaning, and the efforts to create institutional settlements as well as to destabilize, dismantle, and reconfigure them. All are important moments in the ways of the social world. (Scott, 2010: 17)

Of the many approaches that have been devised to construct frameworks for understanding social change, Scott draws together some key features that are distinctive to an institutional perspective:

- A detailed attention to existing institutional variety at the organisation, sector field, or societal level;

- A focus on the interdependence of and interactions between organised units at multiple levels;

- An awareness of the effects of non-local, as well as local factors;

- An appreciation of the temporal nature of social life, the ongoing effects of the past on the present and future;

- emphasis on the importance of ideas (symbolic systems) that mediate between environmental conditions and actions;

- an awareness that processes may produce convergence of procedures and forms but also promote diversity and the emergence of new types of social behaviour and novel systems.

Scott concludes that: 
Important changes are proceeding apace throughout the developed and developing world, and while there are signs of increasing homogenization and convergence, equally strong indicators attest to the preservation and development of diversity. (2010: 18)

It is therefore a diverse and inclusive postdigital debate that universities are in a unique position to lead on and institutional theory has its place in helping to 'tease out and untangle the complex structures involved and the diverse processes and mechanisms at work' (Scott, 2020: 18). This could include taking forward cross-sector debate on possible postdigital scenarios, and their strengths in this context for new approaches towards inclusivity.

\subsection{Postdigital Scenario Planning and Inclusivity}

Matt Finch describes future scenario planning as instructional fables for organisations' (Finch, 2020). With the pace of digitalisation and scientific change that is before us, fables could soon become realities. Therefore, some postdigital scenario planning could help us examine different possible futures that would offer alternative to a neoliberal conception of sustainability and take us in the direction of a post-corporate cultural model of critical sustainability (Delanty, 2020: 4). For example, Michael Peters raises the question:

Are we destined to evolve into bioinformational beings that become more and more integrated into a single evolving data processing system? Once the link between bioinformational technologies and cognitive sciences is made at the nano-level... then, surely, corporations and governments will be able to hack human beings. Goodbye humanism as an educational and pedagogical philosophy. (2020:600)

Here then is a question that has to involve both the disciplines of Humanities and Computing. Finch suggests there are a very broad range of questions that are yet to become clear:

The pandemic has accelerated or triggered numerous transformations whose full consequences are yet to become clear, while other, previously existing trends may now bend or break as a result of 2020's crises. The pandemic response may, in turn, cause new uncertainties - from the long-term health of the economy to the immediate question of university admissions. (Finch, 2020)

However, what remains important is that universities develop new strategic capabilities. This includes: 
rigorous and disciplined approaches to uncertainty which allow us to make leadership decisions on bases other than the evidence and experience of a past which may never repeat. (Finch, 2020)

The use of scenario planning therefore offers:

one method to convene a community of forward-thinking practitioners and engage them in the serious discussion of our strategic blindspots, informing and enriching future decision-making for the post-pandemic university. (Finch, 2020)

University leaders are no exception to the intersecting aspects of positionality discussed earlier by Torres-Olave and Lee (2019). Therefore, in postdigital debate across a range of scenarios there is power to bring together the communities Finch describes to influence our collective futures. It is necessary though for this debate to combine both academic and cross-sector civic leadership if the broad and contentious concerns that fall under inclusivity are to be addressed. The scenarios may sound futuristic but they may also be potentially current circumstances, bringing both complexities but also unintended consequences to plan for. 